\title{
Breast Reconstruction with Microvascular MS-TRAM and DIEP Flaps
}

\author{
David W. Chang \\ Department of Plastic Surgery, The University of Texas MD Anderson Cancer Center, Houston, TX, USA
}

The free muscle-sparing transverse rectus abdominis myocutaneous (MS-TRAM) and deep inferior epigastric perforator (DIEP) flaps involve transferring skin and subcutaneous tissue from the lower abdominal area and have many features that make them well suited for breast reconstruction. The robust blood supply of the free flap reduces the risk of fat necrosis and also enables aggressive shaping of the flap for breast reconstruction to optimize the aesthetic outcome. In addition, the free MS-TRAM flap and DIEP flap require minimal donorsite sacrifice in most cases. With proper patient selection and safe surgical technique, the free MS-TRAM flap and DIEP flap can transfer the lower abdominal skin and subcutaneous tissue to provide an aesthetically pleasing breast reconstruction with minimal donor-site morbidity.

Keywords Free tissue flaps / Mammaplasty / Mastectomy
Correspondence: David W. Chang Department of Plastic Surgery, Unit 443, The University of Texas MD, Anderson Cancer Center,

1515 Holcombe Blvd, Houston, TX 77030, USA

Tel: +1-713-794-1247

Fax: +1-713-794-5492

E-mail: dchang@mdanderson.org

No potential conflict of interest relevant to this article was reported.

\section{INTRODUCTION}

Since it was first described in 1979, the free transverse rectus abdominis myocutaneous (TRAM) flap has become one of the most popular and reliable methods of microsurgical breast reconstruction in breast cancer patients $[1,2]$. Over time, the free TRAM flap has evolved to include the muscle-sparing (MS) TRAM flap and the deep inferior epigastric perforator (DIEP) flap, to minimize donor site morbidity. Both the free MS-TRAM and DIEP flap methods involve transferring skin and subcutaneous tissue from the lower abdominal area to obtain an aesthetically pleasing breast reconstruction.

\section{Indications}

An ideal candidate for breast reconstruction with a free MSTRAM of DIEP flap is a healthy patient who has moderate amounts of abdominal skin laxity and fat and who requires a minimal to moderate volume breast reconstruction. The patient must be willing to undergo the long, complex procedure and to accept the possibility of a prolonged postoperative recovery. She must also understand and accept that she will have an abdominal scar and a potential for donor site morbidities.

\section{Contraindications}

A patient is not a candidate for a free MS-TRAM/DIEP flap if she has an abdominal donor site that cannot be closed primarily because she is too thin or has a potbelly habitus. Other contraindications include a previous TRAM flap or abdominoplasty, a previous abdominal surgery in which the deep inferior epigastric vessels were divided or damaged, or significant medical comorbidities that make the patient a poor surgical candidate.

\section{High-risk patients \\ Smokers}

A patient's history of smoking, by itself, is not an absolute contraindication for microvascular breast reconstruction. We have 
found that free-flap breast reconstruction is not associated with higher rates of vessel thrombosis, flap loss, or fat necrosis in tobacco smokers than in nonsmokers [3]. However, smokers are at significantly higher risk for mastectomy skin flap necrosis, abdominal flap necrosis, and abdominal hernia than are nonsmokers. These smoking-related complications can be significantly reduced when the patient stops smoking at least 4 weeks before surgery.

I recommend a more cautious approach, however, for DIEP flaps because only selected few perforators are included and the perfusion of the flap is not as robust as in free MS-TRAM flaps. For smokers, a safer approach is to optimize the perfusion to the flap by incorporating multiple perforators, thus minimizing fat necrosis and other flap-related complications. Therefore, a free MS-TRAM flap is recommended for smokers, and it is safer to avoid a DIEP flap in this group of patients.

\section{Obese patients}

Obese patients have been found to have significantly higher flap and donor-site complications than patients who have body mass index scores in the healthy range [4]. Specifically, compared with normal-weight patients, obese patients have significantly higher rates of total flap loss, flap seroma, mastectomy skin flap necrosis, abdominal hernia, donor-site infection, and donor-site seroma. In fact, there appears to be an almost linear relationship between complications of all kinds and body weight.

As in smokers, a safer approach for obese patients is to optimize the perfusion to the flap by incorporating multiple perforators with the use of a free MS-TRAM flap, thus minimizing fat necrosis and other flap-related complications.

\section{Previous abdominal suction-assisted lipectomy}

In patients who have undergone a previous abdominal suctionassisted lipectomy (SAL) procedure, the obvious concern is that the perforating vessels to the flap and the microvasculature to the flap may have been damaged, which could compromise the viability of the flap. A few cases of free TRAM flap breast reconstruction following SAL of the abdomen have been reported [5]. When in question, preoperative Doppler ultrasonography or computed tomography angiography can be used to confirm the presence and the patency of the perforating vessels of the abdominal wall. Additionally, the surgeon should consider incorporating a maximum number of perforators into the flap to render it more robust for transfer.

\section{PREOPERATIVE PLANNING}

\section{Patient evaluation}

Free-flap procedures impose major surgical stress on the patient.
Two simultaneous operative sites cause considerable fluid loss, and patients tend to become hypothermic because of the lengthy nature of these procedures. Thus, candidates for free-flap reconstruction must have their cardiac, pulmonary, and renal statuses carefully evaluated preoperatively $[6,7]$.

Patients must be advised to abstain from smoking for at least 4 weeks prior to surgery to reduce the risks of anesthetic complications and wound-healing problems. It is also important for patients to avoid aspirin-containing products for 2 weeks before surgery so that the baseline coagulation status is normal.

To ensure that the patient is a good candidate for this procedure, the patient's abdomen should be evaluated. In particular, the abdomen should be examined for scarring. If there are scars, their location, length, duration, and cause must be considered to determine whether a free abdominal flap procedure can be performed safely [8]. The abdomen should be examined with the patient in a supine position with the knees flexed to ensure that the abdomen can be closed primarily after the harvesting of the abdominal flap. In addition, the integrity of the abdominal wall must be examined for the presence of hernias and for a potbelly habitus.

Once it is determined that the patient is a good candidate for a free abdominal flap, the design of the flap is marked with the patient standing. The inframammary folds are marked bilaterally. The abdominal flap is designed in the lower abdomen with a transverse skin flap. The upper marking is usually just at or above the umbilicus, and the lower marking is just above the pubis, generally following the natural skin fold there. The design of the flap is then tapered to the anterior superior iliac spine so that closure of the donor site will not result in a dog-ear.

\section{Adjuvant therapy}

When considering breast reconstruction for breast cancer patients who need adjuvant therapy, surgeons must take into account the potential effects of breast reconstruction on the adjuvant therapy, and vice versa. The safety, efficacy, and timing of breast reconstruction in patients who require adjuvant therapy must be evaluated to ensure that reconstruction does not delay adjuvant therapy or negatively affect the disease-free interval or overall survival. Thus, the effect of adjuvant treatment on the approach and overall outcome of breast reconstruction merits clarification.

\section{Chemotherapy}

Generally, neoadjuvant chemotherapy is not a contraindication to immediate breast reconstruction and does not increase the complication rate or substantially delay further adjuvant therapy. At our institution, we recommend delaying reconstruction for 3 to 4 weeks following neoadjuvant chemotherapy to allow 
the immunosuppressive effects of the chemotherapy to resolve.

Whether delaying adjuvant chemotherapy affects cancer-related outcomes is not yet definitively known, but most oncologists prefer to initiate therapy within 4 to 6 weeks after the mastectomy or breast-conservation surgery because of concerns that a longer delay may increase recurrence or diminish survival rate. Immediate breast reconstruction does not affect overall survival and recurrence rates.

\section{Hormone therapy}

Despite the benefits of tamoxifen, $1 \%$ to $2 \%$ of patients using it may experience thromboembolic events such as deep vein thromboses, pulmonary embolisms, and cerebrovascular thrombi. Because tamoxifen presents a theoretical risk of thrombosis, it may be appropriate to have the patient stop tamoxifen therapy 10 to 14 days prior to undergoing free-flap reconstruction. After consulting with the patient's medical and surgical oncologists to confirm that tamoxifen therapy can be stopped safely without negatively affecting the patient's cancer treatment, tamoxifen can be stopped and restarted after breast reconstruction.

\section{Radiotherapy}

For some surgeons, immediate reconstruction with autologous tissue remains the preferred approach in patients who require adjuvant radiotherapy. However, many feel that irradiating a reconstructed breast may diminish the aesthetic outcome and thus advocate delaying reconstruction in patients who require adjuvant radiotherapy $[9,10]$. Also, delaying reconstruction until after radiotherapy decreases the risks of fat necrosis, volume loss, and the need for additional flaps. Furthermore, immediate reconstruction may cause technical problems when designing the radiation fields necessary to deliver adjuvant radiotherapy.

\section{SURGERY}

\section{Positioning}

The patient should be placed in a supine position, lying symmetrically and straight on the table. Her waist should be positioned at the proper bend of the table so that she can be placed in a sitting position during flap insetting and shaping. To place the patient in a sitting position, most operating tables need to be reversed so that the patient's head is at the foot of the table. The patient's arms are extended outward and placed on arm boards that have ample foam padding at the elbows and wrists. This allows the breast surgeons to have access to the axilla if lymph node dissection is needed. For immediate reconstruction, the flap harvest and the breast resection are accomplished simultaneously to reduce operating time. For delayed reconstruction, the recipient site preparation and the flap harvest can be performed simultaneously by two teams of surgeons.

\section{Flap harvesting technique}

All flap and recipient site dissection is done under loupe magnification. The border of the skin island is incised down to the abdominal wall. The superficial inferior epigastric vein (SIEV) and superficial inferior epigastric artery (SIEA) are identified. If the SIEA is of significant size and the patient is a good candidate, a SIEA flap can be used for breast reconstruction. Even if a SIEA flap is not planned, it is important to preserve and dissect out the SIEV for about 4 to $5 \mathrm{~cm}$ in length. With the increasing use of DIEP and free MS-TRAM flaps with fewer perforators being included with the flap, occasionally the SIEV needs to be used as a secondary means of venous drainage if deep venous drainage alone is not adequate.

\section{MS-TRAM flap}

With the MS-TRAM flap, the skin and subcutaneous tissues are elevated off the anterior rectus sheath from lateral to medial until the lateral perforators are seen. At this time, lateral perforators from both the right and left sides are evaluated, and the decision is made as to which perforators will be included in the flap. The size, number, and orientation of the perforators should be considered when making this evaluation. The lateral perforators that will not be used are hemoclipped and divided, and then the flap is elevated to expose the medial perforators. Once again, perforators on both sides are evaluated, the preferred perforators are kept, and the others are hemoclipped and divided. This maneuver is continued until two or more of the best perforators remain and have been selected for inclusion in the flap. With all things being equal, the author prefers medially located perforators on the side of the abdomen contralateral from the breast defect. Medial perforators provide longer pedicle length and less functional damage to the remaining rectus muscle since the muscle is innervated from lateral to medial. Also, compared with the laterally located perforators, medially located perforators provide better perfusion to the tissues across the midline of the flap. Generally, two or three moderate to large perforators will provide sufficient circulation to the MS-TRAM flap in most cases.

A fascia-sparing technique is used to open the rectus sheath fascia, incorporating only a small cuff of fascia around the perforators and then connecting these islands of fascia to each other. When the fascia is opened in this manner, only a minimal amount of fascia is sacrificed, facilitating primary closure of the fascia without tension or use of synthetic mesh. The rectus sheath incision is extended inferiorly and laterally to expose the underlying rectus abdominis muscle. 
The anterior rectus sheath fascia is dissected off the underlying rectus abdominis muscle and its inscriptions, and the orientation and course of the perforators within the rectus muscle are evaluated. The decision to perform an MS-TRAM or a DIEP flap procedure is made based on the number, caliber, and location of perforators as well as their orientation and course within the rectus muscle. If the perforators are located in different intramuscular layers, the muscle fibers between the perforators would need to be divided for a DIEP flap; under these circumstances, a small cuff of muscle fibers between and around the perforators is incorporated, and a free MS-TRAM flap is performed (Fig. 1).

Various types of free MS-TRAM flaps can be performed, for the most part, depending on the location and orientation of the perforators. The medial portion of the rectus abdominis muscle can be preserved and a lateral portion of the rectus muscle harvested with the flap (MS-1M). A lateral portion of the muscle can be preserved and the medial portion of the rectus muscle harvested with the flap (MS-1L). Finally, a small cuff of muscle around the perforators can be harvested with the flap, leaving the majority of the muscles intact (MS-2).

Also, the orientation of the perforators within the rectus muscle is a determining factor in how much of the rectus abdominis muscle will be taken with the perforators. If the perforators are coming directly up through the muscle, then only a small amount of muscle needs to be sacrificed. However, if the perforators are coursing obliquely through the muscle, then more muscle will need to be sacrificed. After the flap is harvested, the muscle is secured to the overlying flap with several sutures to minimize any undue tension or twisting of the perforators.

\section{DIEP flap}

If the perforating vessels alone are harvested, sparing the entire rectus abdominis muscle, the resulting flap is referred to as a

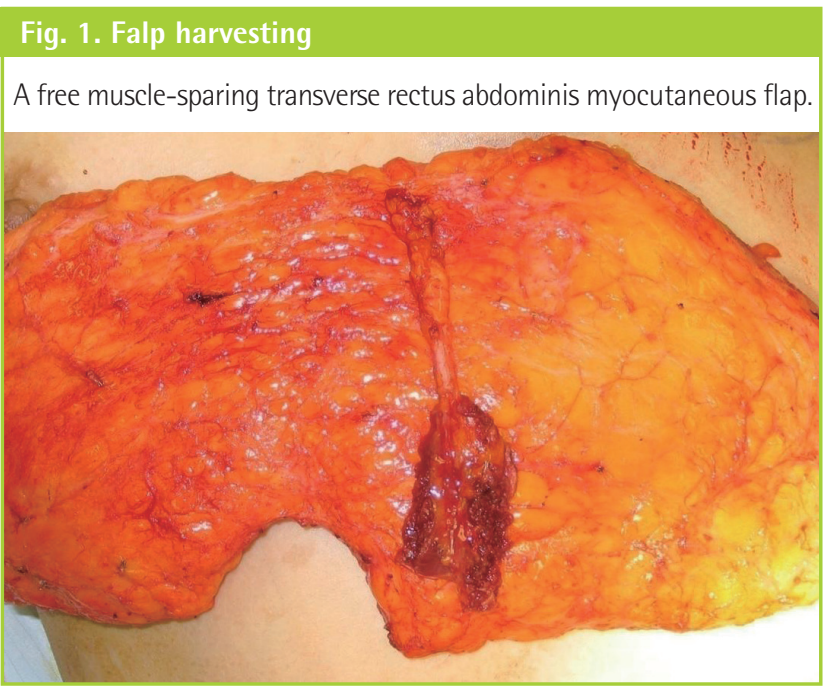

DIEP flap (Fig. 2). Sparing the entire muscle potentially reduces donor-site morbidity, including abdominal bulge and weakness. However, whether use of DIEP flaps reduces these complications significantly more than does use of free MS-TRAM flaps has not been clearly established.

The flap is elevated as described for the free MS-TRAM flap. A DIEP flap is selected ideally when there is a single large perforator or when two or more perforators are located within the same intramuscular septum. The optimal situation for a DIEP flap is when it can be harvested without significant damage to the rectus abdominis muscle.

Once the perforators are identified, each perforator is followed down into the intramuscular septum. The muscle is then split bluntly and sharply and the perforators are dissected to the main branch. The main branch is then followed until the deep inferior epigastric pedicle is visualized. In an ideal condition for a DIEP flap, there is a minimal need for cutting or resecting the rectus muscle.

\section{Preparation of the recipient site}

For immediate reconstruction, after the mastectomy is completed, the mastectomy skin flap and the mastectomy defect are carefully evaluated before the recipient vessels are dissected. For delayed reconstruction, the previous mastectomy scar is excised and sent for pathologic evaluation.

Currently, the author uses the internal mammary vessels as the primary recipient vessels of choice [11-14]. The intercostal spaces are palpated to find an optimal space that is wide and readily accessible for comfortable microvascular anastomoses. This space is usually at the second or third intercostal space. Occasionally, fairly large perforator vessels can be seen medially coming out of the pectoralis muscle fibers. Usually, perforating veins are large and have a very thin wall, and perforating arteries are small.

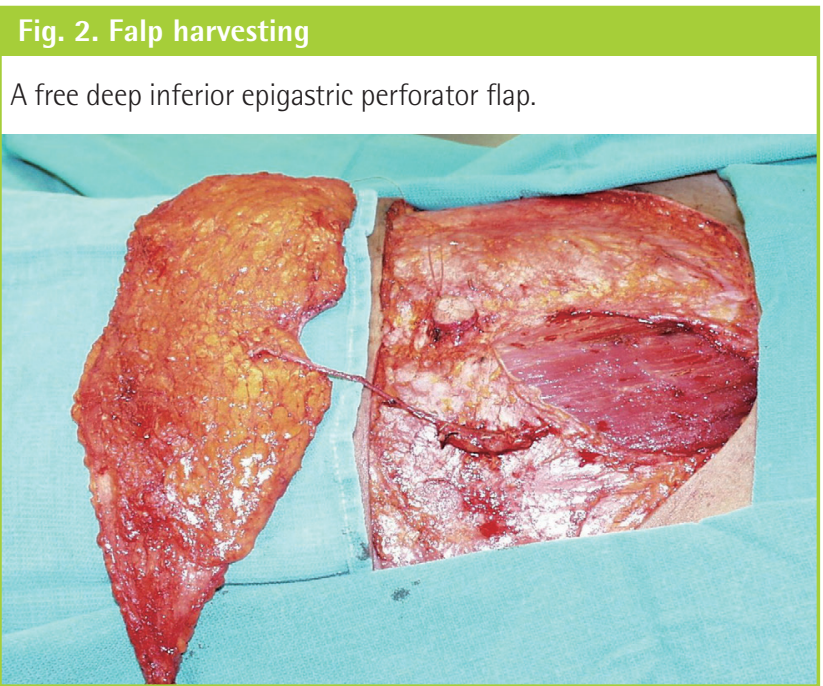




\section{Fig. 3. The recipient site}

An exposure of the internal mammary vessels. $U_{1}$ up; $L_{\text {, lateral; } M_{1}}$ medial; IMA, internal mammary artery; IMV, internal mammary vein.

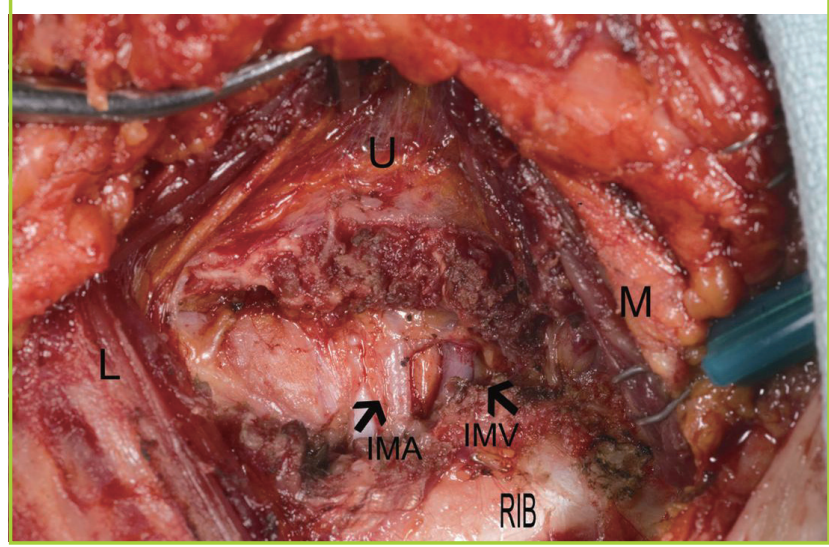

Also, most perforators will pose a size mismatch with the main deep inferior epigastric artery and deep inferior epigastric vein.

The pectoralis muscle at the desired intercostal space is split in the direction of its fibers to expose the intercostal space. There is no need to detach the pectoralis muscle from the sternum. Usually, more perforators can be seen underneath the pectoralis muscle, coming out of the intercostal muscles. Again, the perforators are evaluated for their suitability, and if the surgeon feels they are large enough, then they can be used as recipient vessels.

To expose the internal mammary vessels, the overlying intercostal muscle fibers are carefully divided layer by layer with a bipolar device. Usually, within 1 to $3 \mathrm{~cm}$ from the sternal edge, the internal mammary vein and artery are identified (Fig. 3). Adjacent cartilage does not need to be routinely removed; however, if the intercostal space is narrow or deep, making anastomoses difficult, then the cartilage above or below should be removed to provide better exposure of the vessels. To accomplish this, the perichondrium is incised and dissected off of the cartilage, 2 to $3 \mathrm{~cm}$ of cartilage is removed directly over the internal mammary vessels using a rongeur, and then the perichondrium is carefully dissected off the internal mammary vessels. Once again, extreme care must be taken to control bleeding from all small branches. Final preparation of the recipient vessels is best performed under a microscope.

\section{Microvascular anastomoses}

The flap is secured to the chest wall with sutures or staples, and the vascular pedicle is aligned for anastomoses. It is important to ensure that the vascular pedicle is not twisted prior to performing the microvascular anastomoses. Usually one arterial and one venous anastomoses are sufficient.

\section{Flap insetting and shaping}

The author prefers to place the flap in a vertical fashion for breast reconstruction. This is best accomplished by using a flap from the contralateral side of the abdomen, which allows the flap to lie vertically on the chest with the vascular pedicle oriented medially, in an anatomically natural position, toward the internal mammary recipient vessels. The SIEV will also be oriented medially, so if a second source of venous drainage is needed the SIEV can be anastomosed to an internal mammary perforator vein or to a second internal mammary vein. The thin portion of the flap is inset in the superior region, and the thickest portion of the flap is at the mound of the reconstructed breast. The zone 4 of the flap is always discarded.

During the insetting and the shaping of the flap, the surgeon must always be aware of the tension, the rotation, and the status of the vascular pedicle. Some surgeons like to secure the flap to the chest wall, but it is unnecessary in most cases, and the sutures may create an unnatural contour of the reconstructed breast. Usually, the mastectomy skin flap alone will provide good support for the flap. However, when the flap is significantly smaller than the mastectomy defect, the flap needs to be secured medially and superiorly so it does not fall down within the pocket, which can cause excessive tension on the vascular pedicle.

When shaping a flap for breast reconstruction, it is important to shape the breast with the patient in a sitting position to obtain a natural shape and to focus on the superior and medial areas of the breast to ensure adequate cleavage volume. A slightly overcorrected cleavage region can be easily revised with SAL. However, undercorrected cleavage resulting from deficient tissue volume in the superior and medial portions of the breast can be difficult to correct. It is also better to make the initial breast mound volume slightly larger than the contralateral breast, as a slightly too-large breast can usually be readily revised with SAL or direct excision. Also, the surgeon must ensure that the inframammary fold is placed in the correct location since it is very difficult to adjust later on. Once the optimal size and shape have been achieved, the skin paddle is marked. The patient is then returned to a supine position, and the buried portion of the skin is deepithelialized. One drain is placed underneath the flap.

In unilateral breast reconstruction, the contralateral native breast is used as a guide to achieve volume and shape symmetry for the reconstructed breast. When immediate reconstruction is performed following a skin-sparing mastectomy, the remaining mastectomy skin envelope can facilitate the shaping of the flap for breast reconstruction. In delayed breast reconstruction, the surgeon must decide how to manage the inferior portion of the mastectomy skin flap. If the skin flap is abundant and soft, the author prefers to preserve it and use it for the breast reconstruc- 


\section{Fig. 4. Shaping of delyed breast reconstruction}

(A) Preoperative marking. (B) Delayed reconstruction with a free muscle-sparing transverse rectus abdominis myocutaneous flap.
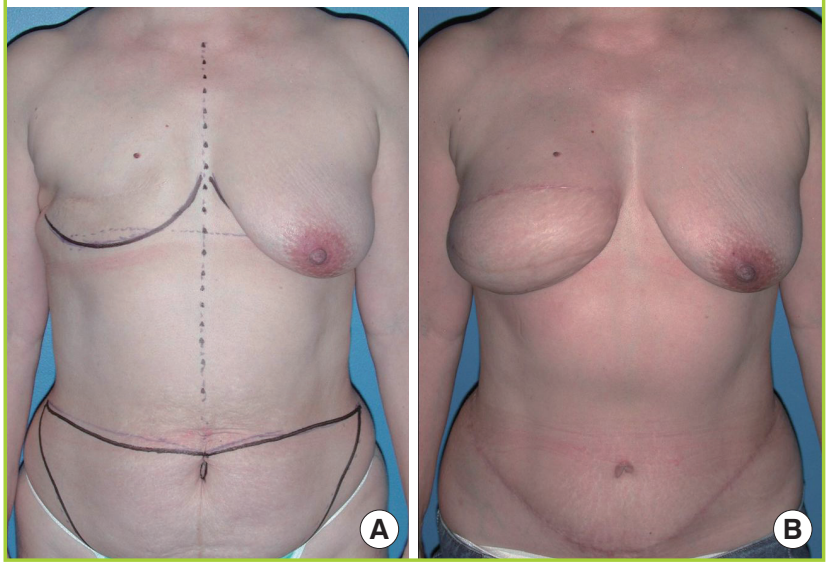

tion, as this allows for a more "natural" shape and appearance (Fig. 4).

One of the most difficult parts of delayed breast reconstruction is achieving an optimal inframammary fold. A careful and accurate preoperative marking with the patient in a standing position is essential in creating an inframammary fold in the proper location. An inframammary fold that is created too high or too low during the initial reconstructive procedure is often difficult to correct. However, it is better to err on the side of making the fold slightly too high rather than too low. The author personally finds it easier to lower an inframammary fold that is too high than to raise an inframammary fold that is too low.

\section{Management of the donor site}

Meticulous closure of the donor site defect is required to prevent a weakening or herniation of the anterior abdominal wall. If the fascia was harvested using a fascia-sparing technique, the medial and lateral cuffs of the anterior rectus sheath can be closed primarily where it was split longitudinally without creating any tension, even in cases of a bilateral flap harvest. The rectus fascia sheath is closed primarily with nonabsorbable sutures using an interrupted and running technique (Fig. 5). Closed-suction drains are placed above the fascia closure in the subcutaneous tissue.

After the abdomen is closed, the umbilicus is brought out through an incision made in the midline of the abdominal flap and secured with sutures. Care should be taken to ensure that the umbilicus is in the correct anatomic location in the midline and that the umbilicus is not twisted at its stalk. There are many different ways to inset the umbilicus. I prefer to make a "frown" incision and to resect a small wedge from the inferior portion of the umbilicus for a more youthful-appearing umbilicus.
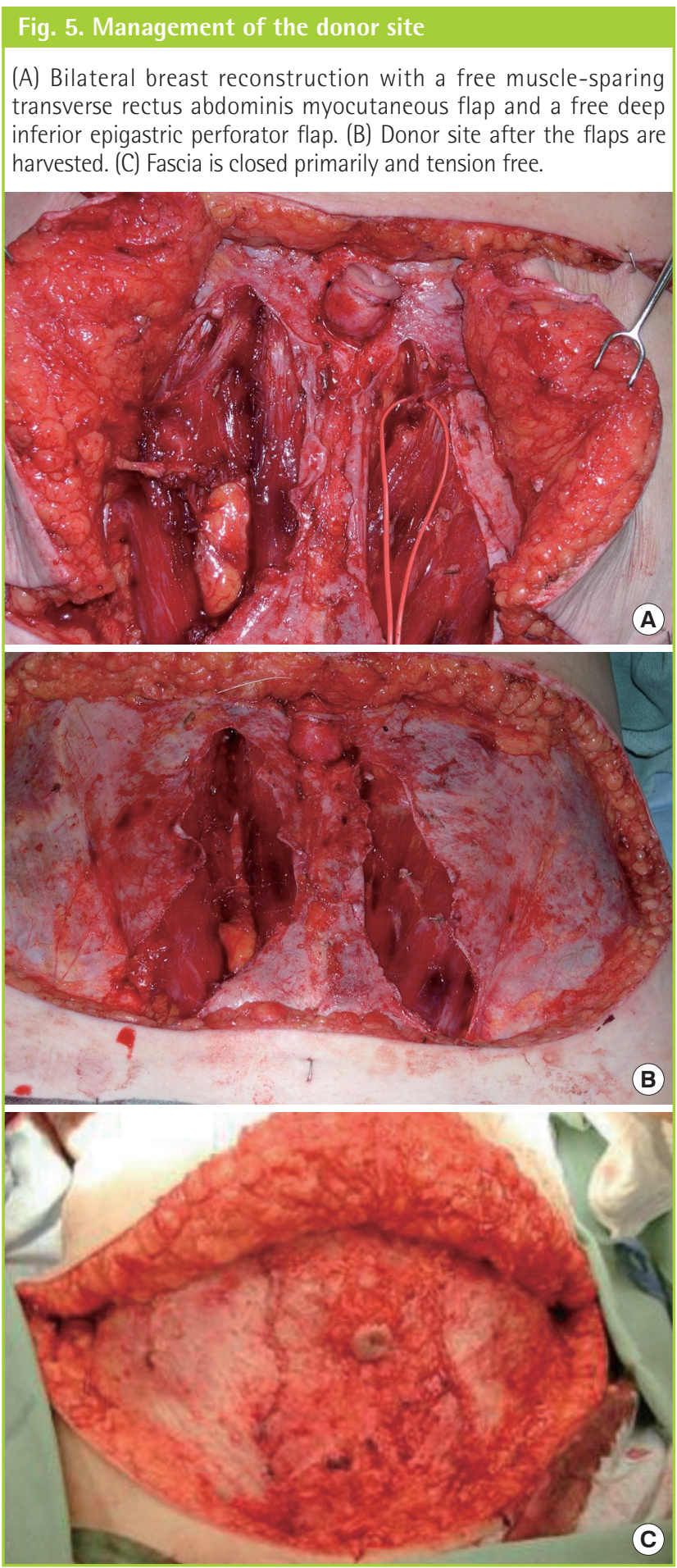

\section{POSTOPERATIVE CARE}

\section{Recovery}

For most cases, no special vasodilators or anticoagulation agents are needed after surgery. A support bra is placed on the patient to help support and maintain the position of the reconstructed breast medially. The patient is placed in a flexed position to mini- 
mize tension at the abdominal donor site, and the flap is monitored every hour by the nursing and/or surgical staff. The patient must refrain from oral intake on the day of surgery. The next morning, if the flap is healing well, the patient's diet is advanced as tolerated. Early postoperative ambulation is encouraged. Exercise that involves the abdomen may be resumed approximately 6 weeks following surgery.

\section{Revisions}

During the initial breast reconstruction, it is often not possible to make a breast that exactly matches the size and shape of the opposite breast. Thus, it is reasonable to expect that the final product will need some touch-ups in many cases. The extent of revision surgery can vary from minor outpatient surgery with local anesthesia to major intervention requiring general anesthesia. It is best to wait until the reconstructed breast has healed and the final volume and shape have been achieved before performing any revision. I usually waits at least 3 months before doing any revisions.

\section{COMIPICATIONS}

\section{Flap loss}

The most common cause of vessel thromboses leading to flap loss is technical error during the microvascular anastomoses. Thus, it is critical that the surgeon have a thorough understanding of the physiologic factors that affect anastomotic patency, technical competence, and sound clinical judgment gained from experience.

\section{Fat necrosis/Partial flap loss}

Fat necrosis and partial flap loss result from inadequate perfusion to a portion of the flap. To minimize fat necrosis and partial flap loss, the surgeon should ensure that perfusion to the flap is optimal and that any poorly perfused area of the flap is discarded, including any areas of the flap that do not have bright red bleeding. In almost all cases, zone 4 tissue should be discarded.

In many cases, two or three perforators-and occasionally even a single, large perforator-provide sufficient perfusion to a flap. However, in high-risk patients, such as those who smoke or are obese, the surgeon should consider including more perforators to reduce the risk of significant fat necrosis or partial flap loss.

Proper selection of recipient vessels is also important to ensure optimal blood inflow and outflow. It is elegant to use perforators as recipient vessels, but the surgeon must be careful to consider the vessel size match and the size of the flap being used for breast reconstruction to minimize complications.

\section{Fig. 6. Case}

(A) A patient with left breast cancer. (B) A long term result following immediate left breast reconstruction with a free deep inferior epigastric perforator flap and subsequent nipple reconstruction. The patient tattooed her donor site scar.
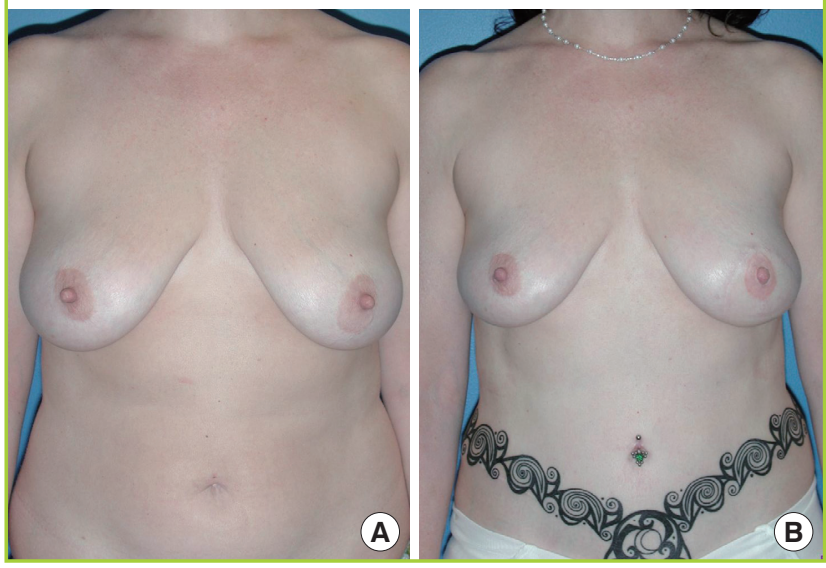

\section{Abdominal bulge/Hernia}

To minimize abdominal donor-site morbidity, particularly hernia or bulge, it is important to obtain optimal tension-free fascial closure $[15,16]$. When a fascia-sparing technique is used in the harvesting of a free MS-TRAM or a DIEP flap, minimal rectus fascia is sacrificed regardless of how much rectus abdominis muscle is sacrificed. Thus, even in bilateral cases, the rectus fascia sheath can be closed primarily with minimal tension.

\section{CONCLUSIONS}

The free MS-TRAM flap and DIEP flap have many features that make them well suited for breast reconstruction. Most patients have adequate lower abdominal skin and subcutaneous tissue available for incorporation into the flap to reconstruct a breast. Its vascular pedicle is large, long, constant, and reliable. The robust blood supply of the free flap reduces the risk of fat necrosis and also enables aggressive folding, trimming, and shaping of the flap for breast reconstruction to optimize the aesthetic outcome. In addition, the free MS-TRAM flap and DIEP flap require minimal donor-site sacrifice in most cases.

With proper patient selection and safe surgical technique, the free MS-TRAM flap and DIEP flap can transfer the lower abdominal skin and subcutaneous tissue to provide an aesthetically pleasing breast reconstruction with minimal donor-site morbidity (Fig. 6).

\section{REFERENCES}

1. Holmstrom $\mathrm{H}$. The free abdominoplasty flap and its use in breast reconstruction: an experimental study and clinical 
case report. Scand J Plast Reconstr Surg 1979;13:423-27.

2. Roehl KR, Baumann DP, Chevray PM, et al. Evaluation of outcomes in breast reconstructions combining lower abdominal free flaps and permanent implants. Plast Reconstr Surg 2010;126:349-57.

3. Chang DW, Reece GP, Wang B, et al. Effect of smoking on complications in patients undergoing free TRAM flap breast reconstruction. Plast Reconstr Surg 2000;105:2374-80.

4. Chang DW, Wang B, Robb GL, et al. Effect of obesity on flap and donor-site complications in free transverse rectus abdominis myocutaneous flap breast reconstruction. Plast Reconstr Surg 2000;105:1640-8.

5. Kim JY, Chang DW, Temple C, et al. Free transverse rectus abdominis musculocutaneous flap breast reconstruction in patients with prior abdominal suction-assisted lipectomy. Plast Reconstr Surg 2004;113:28e-31e.

6. Lipa JE, Youssef AA, Kuerer HM, et al. Breast reconstruction in older women: advantages of autogenous tissue. Plast Reconstr Surg 2003; 111:1110-21.

7. Miller RB, Reece G, Kroll SS, et al. Microvascular breast reconstruction in the diabetic patient. Plast Reconstr Surg 2007;119:38-45.

8. Heller L, Feledy JA, Chang DW. Strategies and options for free TRAM flap breast reconstruction in patients with midline abdominal scars. Plast Reconstr Surg 2005;116:753-9.

9. Tran NV, Chang DW, Gupta A, et al. Comparison of immediate and delayed free TRAM flap breast reconstruction in patients receiving postmastectomy radiation therapy. Plast Reconstr Surg 2001;108:78-82.

10. Chang DW, Barnea Y, Robb GL. Effects of an autologous flap combined with an implant for breast reconstruction: an evaluation of 1000 consecutive reconstructions of previously irradiated breasts. Plast Reconstr Surg 2008;122:356-62.

11. Chang DW, Kim S. Breast reconstruction and lymphedema. Plast Reconstr Surg 2010;125:19-23.

12. Saint-Cyr M, Youssef A, Bae HW, et al. Changing trends in recipient vessel selection for microvascular autologous breast reconstruction: an analysis of 1483 consecutive cases. Plast Reconstr Surg 2007;119:1993-2000.

13. Saint-Cyr M, Chang DW, Robb GL, et al. Internal mammary perforator recipient vessels for breast reconstruction using free TRAM, DIEP, and SIEA flaps. Plast Reconstr Surg 2007; 120:1769-73.

14. Sacks JM, Chang DW. Rib-sparing internal mammary vessel harvest for microvascular breast reconstruction in 100 consecutive cases. Plast Reconstr Surg 2009;123:1403-7.

15. Bajaj AK, Chevray PM, Chang DW. Comparison of donorsite complications and functional outcomes in free musclesparing TRAM flap and free DIEP flap breast reconstruction. Plast Reconstr Surg 2006;117:737-46.

16. Wu LC, Bajaj A, Chang DW, et al. Comparison of donorsite morbidity of SIEA, DIEP, and muscle-sparing TRAM flaps for breast reconstruction. Plast Reconstr Surg 2008; 122:702-9. 